\title{
Motion Control for Autonomous Tugger Vehicles in Dynamic Factory Floors Shared with Human Operators*
}

\author{
Luis Louro \\ dept. of Industrial Electronics \\ University of Minho \\ Portugal \\ llouro@dei.uminho.pt \\ Sergio Monteiro \\ dept. of Industrial Electronics \\ University of Minho \\ Portugal \\ sergio@dei.uminho.pt
}

\author{
Tiago Malheiro \\ dept. of Industrial Electronics \\ University of Minho \\ Portugal \\ tmalheiro@dei.uminho.pt
}

\author{
Pedro Guimaraes \\ dept. of Industrial Electronics \\ University of Minho \\ Portugal \\ b7981@algoritmi.uminho.pt
}

Pedro Vaz Silva

$\mathrm{CM} / \mathrm{LOI}-\mathrm{Brg}$

Bosch Car Multimedia

Braga, Portugal

Pedro.Vaz.Silva@pt.bosch.com wolfram.erlhagen@math.uminho.pt
Wolfram Erlhagen

dept. Mathematics and Applications

University of Minho

Portugal

\author{
Toni Machado \\ $\mathrm{CM} / \mathrm{LOI}-\mathrm{Brg}$ \\ Bosch Car Multimedia \\ Braga, Portugal \\ Toni.Machado@pt.bosch.com
}

Estela Bicho**

dept. of Industrial Electronics

University of Minho

Portugal

estela.bicho@dei.uminho.pt

\begin{abstract}
We present a motion controller that generates collision free trajectories for autonomous Tugger vehicles operating in dynamic factory environments, where human operators may coexist. The controller is formalized as a dynamic system of path velocity and heading direction, whose vector fields change as sensory information varies. By design the parameters are tuned so that the control variables are close to an attractor of the resultant dynamics most of the time. This contributes to the overall asymptotically stability of the system and makes it robust against perturbations. We present several experiments, in a real factory environment, that highlight different innovative features of the navigation system - flexible and safe solutions for human-aware autonomous navigation in dynamic and cluttered environments. This means, besides generating online collision free trajectories between via points, the system detects the presence of humans, interact with them showing awareness of their presence, and generate adequate motor behavior.
\end{abstract}

Index Terms-Tugger vehicles, flexible and safe autonomous navigation, obstacle avoidance, dynamic environments shared with human operators

\section{INTRODUCTION}

Efficient material flow within a facility is a key feature for the success of the industrial organizations. It implies that raw materials should be delivered on time, i.e., whenever determined by the production plan, and also finished goods should be picked up on time. When there are several pick and drop locations in the same facility, and they are far from the storage locations (as happens in production lines), it is usual to use vehicles to transport the materials. Typical examples

\footnotetext{
*This work has been supported by National Funds through FCT - Fundação para a Ciência e Tecnologia within the Project Scope: UID/CEC/00319/2019, and by European Structural and Investment Funds in the FEDER component, through the Operational Competitiveness and Internationalization Programme (COMPETE 2020) [Project $n^{\circ}$ 002814; Funding Reference: POCI-01-0247FEDER-002814].

**Corresponding author
}

of such vehicles are tuggers and stackers because they allow transporting large quantities of materials or goods. Today, these vehicles are either operated by humans or are Automated Guided Vehicles (AGVs). While the former offer the flexibility of the human operator deliberative skills, the later present the ability of seamless integration with the factory floor and may relieve the operator to higher value tasks.

For some decades, AGVs have been being used with success in industrial scenarios. In the early scenarios, the AVGs relied on a fixed infrastructure - typically embedded wire-path or visual lines or beacons - to guide the vehicles in fixed paths between locations. In the second generation, free-ranging AGVs, taking advantage of localization systems based on laser triangulation [12] and inertial measurement units [11], have been developed relieving the necessity of pre-defined and rigid infrastructure. Nowadays, one is moving towards the development of truly flexible machines by providing them with autonomous navigation behavior and minimal infrastructure dependance [18]. A framework to develop a flexible AGV system, tested in an industrial forklift that encompasses a modular and layered architecture, is shown in [6]. It incorporates an environment representation and can generate plans for a given task. The navigation module is able to react to obstructions by using avoidance behaviors. Recent advances in imaging system technologies together with an increase of the computing power available even in small devices has induced the appearance of solutions that are, almost, vision-based. In this context [4] uses four different visual servo controllers for navigation, guidance, and pallet localization purposes, while [7] uses monocular SLAM for global navigation and visual servoing for steering motions towards the target. In factory floors where human operators share the environment with autonomous vehicles that do not follow exact guide-paths, 
it is of the utmost importance for the safety of operation the existence of modes of interaction between humans and vehicles [14]. These modes should allow the vehicle not only to react in advance to the presence of humans in its way but also to warn those humans of its own behavior. Typical safety solutions make use of safety LiDARs that stop the vehicle in case of an area violation. However, because LiDARs do not distinguish between humans and objects, obstructions created by one or the other are treated in the same way.

In this paper, we develop solutions for safe, flexible and human-aware autonomous navigation for Tugger vehicles operating in dynamic and cluttered environments, where autonomous vehicles and human operators coexist. This means that besides detecting the presence of humans, the vehicles should also interact with them in order to show awareness of their presence and select the appropriate motor behavior. The results presented here are part of a project with Bosch Car Multimedia (Braga, Portugal) that aimed to develop new solutions - for the movement of raw materials and finished goods within the factory plant - that are based on autonomous vehicles capable of navigating the factory's very dynamic floor plant, while interacting with other vehicles and humans. One of the target vehicles were Tugger and trailer assemblies operating a milk-run process (see Fig. 1).

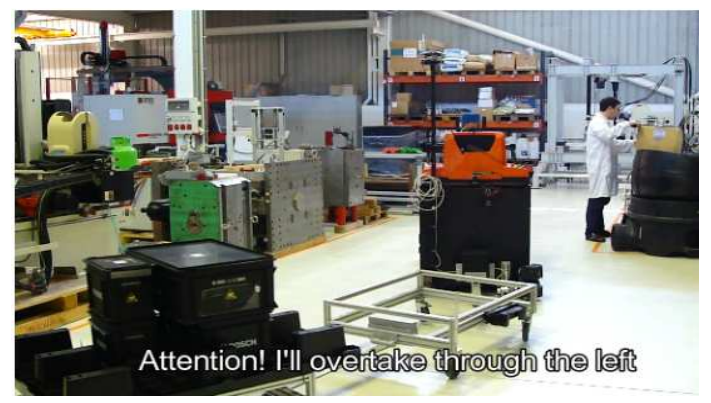

Fig. 1. Tugger and trailer assemblies operating a milk-run process on a dynamic factory floor shared with human operators.

Besides the autonomous navigation system for the Tugger vehicle, which this paper is about, that project also addressed other scientific challenges such as internal logistics planning, scheduling and routing, indoor vehicle localization, and system integration with the plant information systems.

The developed solution for safe, flexible and human aware navigation, is composed of a set of controllers that generate different behaviors (target acquisition, obstacle avoidance, and human avoidance) and methods to select and integrate the behaviors. The approach used is based on the so-called Attractor Dynamics Approach to Behavior Generation [15] and builds on our previous work (e.g., [1], [5], [8]). In the present work, this approach is also a challenge, because to the best of our knowledge it has never been applied to generate the motion of Tugger vehicles. The developed controllers are formalized as nonlinear dynamical systems in terms of path velocity and heading direction, whose vector fields change as sensory information (from both LiDARS and vision systems) varies. By design, the parameters are tuned so that the control variables are close to an attractor of the resultant dynamics most of the time. This contributes to the overall asymptotically stability of the system and makes it robust against perturbations. Furthermore, it is easy to implement and fast to compute, especially when compared to optimization methods [13], [16] (check the above references for some more insights, as the comparison is out of the scope of this paper).

We have tested our motion controllers on simulated and real dynamic factory floors. Results show the ability of the Tugger vehicle (i) to generate on-line collision-free trajectories while moving from point $\mathrm{A}$ to point $\mathrm{B}$, ii) the capacity to react to abrupt perturbations (e.g., the sudden appearance of a box that is dropped by a human operator), (iii) the capacity to avoid human operators that appear on the vehicle's path, and (iv) the capacity to handle human operators that defy mission accomplishment.

The remainder of the paper is structured as follows: section II describes the vehicle hardware and software setup; an overview of the navigation module is presented next, in section III; the dynamics of the motion controller for flexible navigation is presented in section IV; which is followed by the description of how one can use the dynamics to build the communication for human-robot interaction, in section $\mathrm{V}$; implementation details are given in section VI; section VII presents several experimental results, and the paper finishes with conclusions and future work in section VIII.

\section{TUGger VeHICLE}

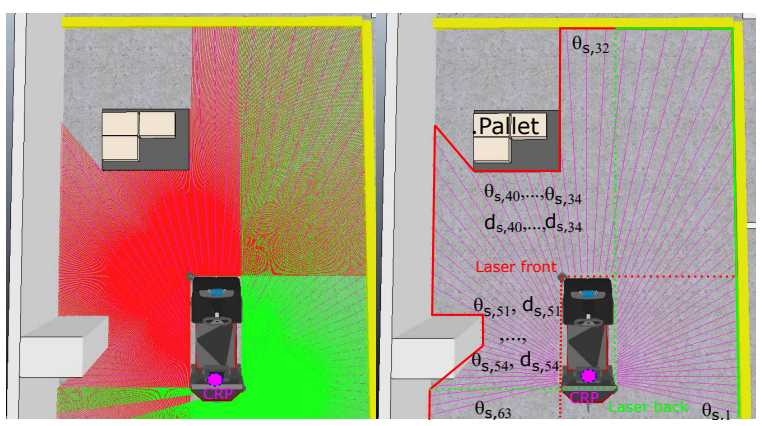

Fig. 2. (a)The two Lidars cover the surrounding space of the vehicle (red-left front lidar; green-right rear Lidar). (b) The space around the vehicle is divided into several sectors centered in the CRP, each with and angular range of $\Delta \theta_{s}$. Sector $i(i=1, \ldots, N$ sectors $)$ is centred at an angle $\theta_{s, i}$ with respect to the vehicle's forward direction, and $d_{s, i}$ is the distance from the periphery of the vehicle to an obstruction sensed at direction $\theta_{s, i}$.

The autonomous Tugger is based on a commercially available human operated tugger machine. To enable autonomous operation, we have installed two safety laser scanners (Sick S300 LiDAR) mounted on vehicle opposite corners to ensure a $360^{\circ}$ vehicle surroundings coverage, see Fig. 2. It secures a hazardous area around the vehicle by triggering the emergency stop should it detect an object or person in the predefined protective fields. The Tugger features a 2D RGB vision sensor which is used to detect humans in the front of the vehicle. In what regards the vehicle locomotion, the 
machine is fitted with two coupled drive systems that control translation and steering (see Fig. 3). These devices and the

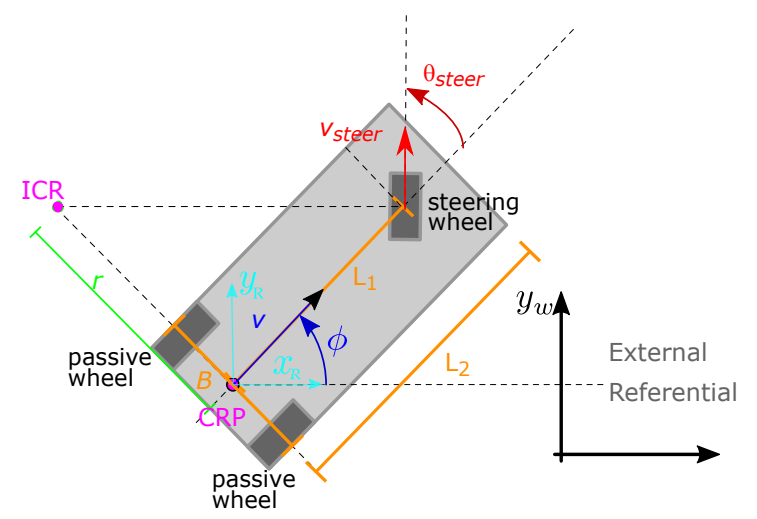

Fig. 3. Tricycle kinematic model for the Tugger vehicle, with actuation variables $\left(\theta_{\text {steer }}\right.$ and $\left.v_{\text {steer }}\right)$ and behavioral variables ( $\phi$-heading direction and $v$ - path velocity).

truck controller (on the tiller arm) are on a Controlled Area Network (CAN). We have developed a dedicated Electronic Control Unit (ECU) to take the CANOpen master function and handle the hard real-time constrains imposed by the drive systems. Furthermore, the ECU manages the analog steering signals, the manual/autonomous driving commutation, the devices power switching, and the safety laser scanners protective fields case switching. The latter is important to adjust the protective fields coverage based on the vehicle's velocity and overall behavior. The developed firmware is based on the Active Object (AO) design pattern [9], and supported by the Quantum Leaps Platform Hierarchical Event Processor (QEP) and the freeRTOS real-time operating system.

The vehicle's actuation is exposed on a ROS network using the ROS Control framework. To this, we have implemented the hardware interfaces (position and velocity interfaces, and joint state interfaces), controllers, and the robot URDF and TF tree.

The vehicle's indoor localization is based on an Inertial Measurement Unit (IMU - xsens MTi-300 AHRS) and a Real-Time Localization System (RTLS - Eliko), providing the vehicle's position $\left({ }^{w} x_{\text {robot }},{ }^{w} y_{\text {robot }}\right)$ and orientation, $\phi$, on the $2 \mathrm{D}$ plan relative to a fixed referential frame. The RTLS applies a technique of multilateration using the times of arrival of the signals between the anchors (fixed to the building) and the tag (attached to the robot) so that a precise 2D indoor position is gathered.

To enable human-robot interaction, we use a ROS based speech synthesis module (sound_play), which allows the Tugger to verbalize its behavior. Furthermore, to support humans detection in the vehicle's navigation path, we have implemented an image processing pipeline with three steps: (1) objects detection, (2) objects tracking and (3) variables estimation. The first step is responsible for detecting and recognizing humans in a frame. This step is supported by the Tensorflow Object Detection API [17], to create and use customized detection models. The second step tracks detected humans over a stream of frames by conferring them an individualized identity. This way, it is possible to know the behavior, e.g. velocity of a certain detected human in the environment during its lifetime in frames. The last step estimates the humans' angle and distance to the vehicle, and humans' velocity in the environment (see, Fig. 4). The algorithm uses the humans'

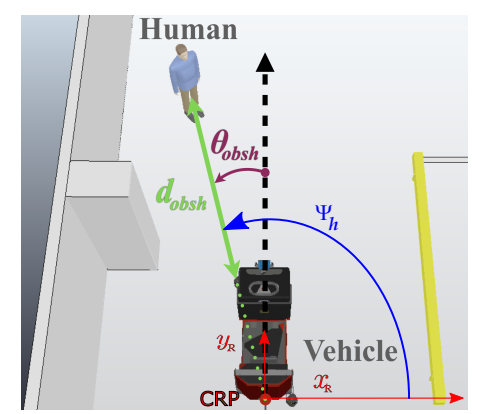

Fig. 4. Vision system tracks detected humans and provides the bearing angle, $\theta_{o b s, h}$. Distance to a detected human $d_{o b s, h}$ is obtained fusing information from the vision system and Lidars.

position in the $2 \mathrm{D}$ RGB image to compute the angle, and fuses it with the laser scanners' data to get the distance to the vehicle. This result is published in a ROS topic.

Finally, it is important to highlight that in our project the trailers and their coupling system have been designed such that the kinematics of the Tugger and trailers chain guarantees that each trolley closely follows the trajectory footprint of the Tugger (details see [10]). The trajectory error between the Tugger and the last trailer is bounded (in our case in the $\mathrm{cm}$ range) and does not depend on the number of trailers [2]. This is very important for two main reasons. First, only the Tugger is equipped with sensors to detect obstructions, and thus it is the only responsible for obstacle avoidance of the chain Tugger+trailers, and second, it greatly simplifies the design of the motion controllers (c.f. section IV).

\section{OVERVIEW OF THE NAVIGATION MODULE}

Fig. 5 shows a block diagram with the various elements that contribute to the navigation module of the vehicle. The Service Manager receives a service from a server (e.g., "Move raw materials to supermarket 1 and then to supermarket 2"), and then, splits the service in a sequence of tasks (e.g., "Task 1 - move to supermarket 1", "Task 2 - move to supermarket 2") that are sent to the Task Manager. The Task Manager splits a task into sequence of targets through which the Tugger has to pass. The coordinates of the next target point, $\left({ }^{w} x_{\text {target }},{ }^{w} y_{\text {target }}\right)$, together with velocity limits, $\left(w_{\max }, v_{\max }\right)$, for the linear and angular velocities in the route to the defined target, are sent to the Navigation module. The Navigation module also receives information from the positioning system and the environment perception, the latter concerning the information of the system of detection of persons and the detection of obstacles. The motor commands generated in the Navigation module are scrutinized by the Safety Monitoring and then sent to the Motor Actions component. The Navigation module also sends 


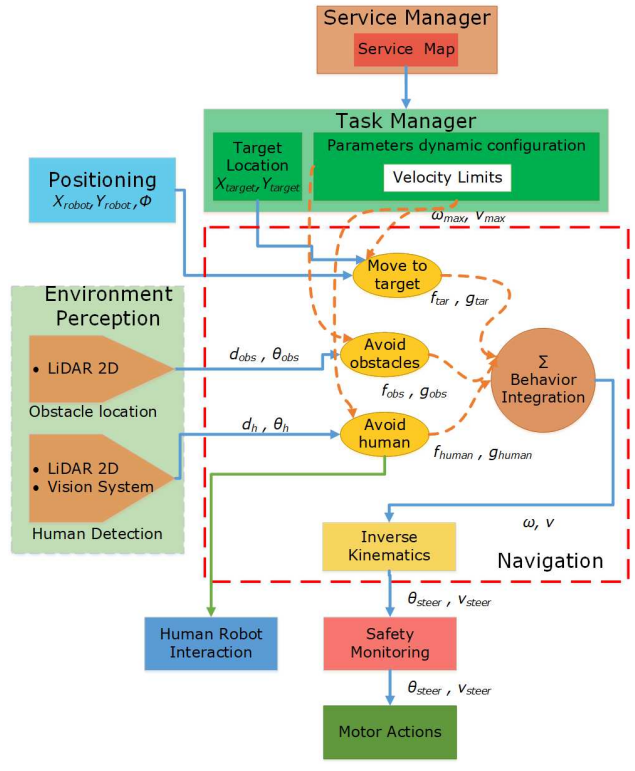

Fig. 5. Blocks diagram for the Navigation module.

text messages to the human-robot interaction module in order to communicate with human operators. This will allow the human operators to know in advance what the Tugger will do, as illustrated, e.g., in Fig. 1 (c.f. also Section VII).

\section{THE DYNAMICS OF FLEXIBLE NAVIGATION}

Flexible navigation means here that the autonomous Tugger must avoid collisions with obstacles or human operators that it may encounter during the execution of its mission.

Motion control is generated from the integration of three basic behaviors: i) move to target via point, ii) avoid obstacles, and iii) avoid humans. Dynamical systems theory is used here as a theoretical language and tool to design each of these behaviors, as well as of their integration. Specifically, to model the motion controller we use the heading direction, $\phi$, and path velocity, $v$, as control/behavioral variables (see Fig. 3). The navigation behavior is generated by providing values in time to these variables, which will then be used to control vehicle's actuated wheel (c.f. section VI). The time course of the behavioral variables, $\phi(t)$ and $v(t)$, is obtained from fixedpoint solutions of dynamical systems in the form of differential equations:

$$
\begin{aligned}
\frac{d \phi}{d t} & =F(\phi, \text { parameters })+f_{\text {stoch }} \\
& =\left(f_{\text {tar }}(\phi)+f_{\text {obs }}(\phi)+f_{\text {human }}(\phi)\right)+f_{\text {stoch }} \\
\frac{d v}{d t} & =G(v, \text { parameters }) \\
& =c_{\text {tar }} g_{\text {tar }}(v)+c_{\text {obs }} g_{\text {obs }}(v)+c_{\text {human }} g_{\text {human }}(v)
\end{aligned}
$$

where the vector fields $F(\phi)$ and $G(v)$ consist of a number of contributions that express independent task constraints (move to target via-point, avoid obstacles, avoid humans). In isolation, each contribution, $f_{i}$ or $g_{i}$ ( $i=t a r, o b s$, human), creates an attractor at a desired value (e.g. direction of the next target via-point) or a repeller at an undesired value (e.g. the direction at which an obstacle or human is detected) with a specified strength and range of attraction or repulsion, respectively. $c_{i}$ ( $i=t a r, o b s$, human) are activation variables that determine the weight of each contribution in Eq. 2. Parameters are tuned such that the motion is governed by a sequence of asymptotically stable states. This makes the control system robust against perturbations or noisy sensory information. The vector field in Eq. 1, which is non-linear, is augmented with a stochastic force, $f_{\text {stoch }}$, to guarantee that the heading direction escapes from repellers, because due to a bifurcation in the vector field (as sensory information varies) it may happen that the attractor in which the variable was sitting becomes a repeller.

Next, we explain how the individual contributions to the vectors fields (1) and (2) are built.

\section{A. Dynamics of heading direction}

The heading direction dynamics (1) has three main components: 1) $f_{\text {tar }}$ is responsible for orienting the vehicle into the direction of the next target via-point; 2) $f_{o b s}$ repels the vehicle's heading direction from directions at which obstructions are signaled; and 3) $f_{\text {human }}$ repels the vehicle's heading direction from the directions at which human operators are detected.

1) Target acquisition: Via-points are defined in external world coordinates, and $\left({ }^{w} x_{\text {target }},{ }^{w} y_{\text {target }}\right)$ represents the next target. The vehicle keeps an estimate of its location $\left({ }^{w} x_{\text {robot }},{ }^{w} y_{\text {robot }}\right)$ in the world reference frame. The direction, $\psi_{\text {tar }}$, relative to the ${ }^{w} x$ world axis in which the target lies is given by $\psi_{\text {tar }}=\tan ^{-1}\left(\left({ }^{w} y_{\text {target }}-{ }^{w} y_{\text {robot }}\right) /\left({ }^{w} x_{\text {target }}-{ }^{w}\right.\right.$ $\left.\left.x_{\text {robot }}\right)\right)$. Rotation towards the direction at which the target point lies is modeled by an attractive force-let

$$
f_{\text {tar }}(\phi(t))=-\lambda_{\text {tar }} \sin \left(\phi(t)-\psi_{\text {tar }}\right)
$$

that puts an attractor fixed point at $\phi=\psi_{\text {tar }}$, with strength of attraction (relaxation rate) defined by $\lambda_{\text {tar }}(>0)$, and a range that covers across the entire $360 \mathrm{deg}$ domain of the heading direction.

2) Collision avoidance with obstacles: An obstacle, either static or dynamic, may give rise to several obstructions being sensed at different sectors (see Fig. 2). Collision avoidance is modeled by:

$$
f_{\text {obs }}(\phi(t))=\sum_{i} f_{o b s, i}(\phi(t))
$$

where $f_{o b s, i}(\phi(t))$ is a repulsive force-let created by an obstruction detected in sector $i$. Each force-let is modeled by:

$$
\begin{aligned}
& f_{o b s, i}(\phi(t))= \\
& =\lambda_{o b s, i}\left(\phi(t)-\psi_{o b s, i}\right) \exp \left(-\frac{\left(\phi(t)-\psi_{o b s, i}\right)^{2}}{2 \sigma_{o b s, i}{ }^{2}}\right)
\end{aligned}
$$

that erects a fixed point repeller at direction $\psi_{\text {obs }, i}$ with strength of repulsion defined by $\lambda_{o b s, i}(>0)$, and with $\sigma_{o b s, i}$ setting the angular range over which the reppeler exerts its effect. The strength of repulsion is given by

$$
\lambda_{o b s, i}=\beta_{1, i}^{o b s} \exp \left(-d_{s, i} / \beta_{2, i}^{o b s}\right)
$$


Here, parameter $\beta_{1, i}^{o b s}$ sets the maximum strength of repulsion created by an obstruction detected in sector $i$, while $\beta_{2, i}^{o b s}$ sets its decay as a function of estimated distance of the obstruction to the periphery of the vehicle:

$$
\begin{gathered}
\beta_{1, i}^{o b s}=\left\{\begin{array}{cc}
k_{1,1}^{o b s} \cdot \exp \left(-\left(\left|\theta_{s, i}\right|-\frac{\pi}{6}\right)\right) & ,\left|\theta_{s, i}\right|>\frac{\pi}{6} \\
k_{1,2}^{o b s} & , \frac{\pi}{12} \leq\left|\theta_{s, i}\right| \leq \frac{\pi}{6} \\
k_{1,3}^{o b s} & , \text { otherwise }
\end{array}\right. \\
\beta_{2, i}^{o b s}=\left\{\begin{array}{cc}
k_{2,1}^{o b s} \cdot \exp \left(-\left(\left|\theta_{s, i}\right|-\frac{\pi}{6}\right)\right) & ,\left|\theta_{s, i}\right|>\frac{\pi}{6} \\
k_{2,2}^{o b s} & , \frac{\pi}{12} \leq\left|\theta_{s, i}\right| \leq \frac{\pi}{6} \\
k_{2,3}^{o b s} & , \text { otherwise }
\end{array}\right.
\end{gathered}
$$

The angular range, $\sigma_{o b s, i}$, over which the repulsive force-let acts also depends on the distance, $d_{s, i}$, to the obstruction in sector $i$ :

$$
\sigma_{o b s, i}=\arctan \left(\tan \left(\Delta \theta_{s} / 2\right)+\frac{B}{2\left(d_{p, i}+d_{s, i}\right)}\right)
$$

where $d_{p, i}$ is the distance of the point in the periphery of the vehicle in direction $\theta_{s, i}$ to the CRP:

$$
d_{\mathrm{p}, i}=\left\{\begin{array}{cc}
\left|\frac{L_{2}}{\cos \left(\theta_{s, i}\right)}\right| & ,\left|\theta_{i}\right|<\tan ^{-1}\left(\frac{B}{2 L_{2}}\right) \\
\left|\frac{B}{2 \cdot \sin \left(\theta_{s, i}\right)}\right| & \text { otherwise }
\end{array}\right.
$$

In the argument of Eq. 9, the first term reflects the angular size of the sector, and the second term expresses the safety margin required for the vehicle to pass next to an obstruction that could occupy maximally the entire sector range. The larger the distance of the obstruction to the CRP $d_{o b s, i}^{C R P}=d_{p, i}+d_{s, i}$, the smaller the angle subtended by a copy of the vehicle if positioned next to the obstruction and viewed from the the actual vehicle's position.

3) Avoidance of human operators: Collision avoidance with human operators in the vicinity of the vehicle is modeled by building repulsive force-lets at the directions, $\psi_{h, i}$ at which they are detected:

$$
f_{\text {human }}(\phi(t))=\sum_{i} f_{\text {human }, i}(\phi(t))
$$

with

$f_{\text {human }, i}(\phi(t))=\lambda_{h, i}\left(\phi(t)-\psi_{h, i}\right) \exp \left(-\frac{\left(\phi(t)-\psi_{h, i}\right)^{2}}{2 \sigma_{h, i}{ }^{2}}\right)$

where the strength of repulsion is given by

$$
\lambda_{h, i}=\beta_{1}^{h} \exp \left(-d_{o b s h, i} / \beta_{2}^{h}\right)
$$

with $\beta_{1}^{h}$ setting the maximal strength of repulsion and $\beta_{2}^{h}$ setting the decay as a function of distance, $d_{o b s h, i}$, to the human operator (Fig. 4). The range of repulsion is given by

$$
\sigma_{h, i}=\arctan \left(\frac{L_{h}+B}{2\left(d_{o b s h, i}+d_{p h, i)}\right.}\right)
$$

where $L_{h}(=80 \mathrm{~cm})$ is the average width of a human adult, and $d_{p h, i}$ is the distance of the point in the periphery of the vehicle, in direction $\theta_{o b s h, i}$, to the CRP (Eq. 10).

\section{B. Dynamics of path velocity}

Each contribution in the path velocity dynamics, Eq. 2, is of the form

$$
g_{i}(v(t))=-\lambda_{v, i}\left(v(t)-v_{d e s, i}\right)
$$

and sets an attractor state at the desired value $v=v_{d e s, i}$ for the vehicle's path velocity, with relaxation rate $\lambda_{v, i}(>0)$ (i=tar, obs, human).

1) Target acquisition: For the target acquisition behavior the attractor for the velocity dynamics is

$$
v_{\text {des }, \text { tar }}=\left\{\begin{array}{cc}
\frac{\left(d_{t a r}^{C R P}-d_{\text {tar }- \text { stop }}^{C R P}\right)}{k_{\text {tar }}^{v}} v_{\max } & , d_{\text {tar }}^{C R P}<D_{\text {tar }} \\
v_{\max } & , \text { otherwise }
\end{array}\right.
$$

where $d_{t a r}^{C R P}$ is the distance of the CRP to the target. While this distance is larger than $D_{\text {tar }}$ the attractor makes the vehicle to drive at the maximum allowed velocity, $v_{\max }$, otherwise as the distance $d_{t a r}^{C R P}$ decreases the attractor shifts toward zero making the vehicle to stop in case the stop distance with respect to the target location, $d_{\text {tar_stop }}^{C R P}$, is reached.

2) Collision avoidance of obstacles: For the obstacle avoidance behavior the desired value for the velocity $v$ is set by

$$
\begin{gathered}
v_{d e s, o b s}= \\
\left\{\begin{array}{cc}
\frac{d_{o b s, \min }}{k_{o b s, 1}^{v}} v_{\max } & , d_{o b s, \min }<D_{o b s, l a t} \\
\frac{\left(d_{o b s, f r o n t}-D_{\text {obs_stop }}\right)}{k_{\text {obs }, 2}} v_{\max } & , d_{o b s, \text { min }, \text { front }}<D_{\text {obs }, \text { front }} \\
0 & , d_{\text {obs }, \text { min }, \text { front }}<D_{\text {obs_stop }} \\
v_{\max } & , \text { otherwise }
\end{array}\right.
\end{gathered}
$$

where $d_{o b s, \min }=\min \left(d_{s, i}\right)(i=1, \ldots, N$ sectors $)$, and $d_{\text {obs,min,front }}$ is the minimum distance among the sectors that point in the front direction of the Tugger, i.e. $d_{o b s, \text { min, front }}=$ $\min \left(d_{s, i}\right)(i=30, \ldots, 34)$. $D_{o b s, l a t}$ and $D_{o b s, \text { front }}$ set the limits below which the Tugger must slow down in case obstructions are sensed in the lateral or front directions, respectively.

3) Avoidance of human operators: For the behavior of avoiding human operators the attractor for path velocity is defined by

$$
v_{\text {des }, \text { human }}=\left\{\begin{array}{cc}
\frac{\left(d_{\text {obsh }}-D_{h_{\_} s t o p}\right)}{k_{h}^{v}} v_{\max } & , d_{\text {obsh }}<D_{\text {obs }, h} \\
0 & , d_{\text {obsh }}<D_{h_{-} s t o p} \\
v_{\max } & , \text { otherwise }
\end{array}\right.
$$

which makes the Tugger to slow down if a human is detected at a distance below $D_{o b s, h}$, coming to rest at a distance $D_{h \_s t o p}$ to the human operator.

4) Activation variables: While for the heading direction dynamics, Eq. 1, all components contribute to the vector field $F(\phi)$, in the path velocity dynamics, Eq. 2, the activation variables determine which contribution should dominate the 
vector field $G(v)$. In the present implementation they are set as follows:

$$
\begin{aligned}
& c_{\text {tar }}=\left\{\begin{array}{cc}
1 & , d_{o b s h} \geq D_{o b s, h} \wedge d_{o b s, \min } \geq D_{o b s, l a t} \wedge \\
& d_{o b s, \text { front }} \geq D_{\text {obs }, \text { front }} \\
0 & , \text { otherwise }
\end{array}\right. \\
& c_{\text {human }}=\left\{\begin{array}{cc}
1 & , d_{\text {obsh }}<D_{o b s, h} \\
0 & \text {, otherwise }
\end{array}\right. \\
& c_{o b s}=c_{o b s}^{\prime}\left(1-c_{\text {human }}\right)
\end{aligned}
$$

with

$$
c_{o b s}^{\prime}=\left\{\begin{array}{cc}
1 & , d_{o b s, \text { min }}<D_{o b s, \text { lat }} \vee d_{o b s, \text { front }}<D_{o b s, \text { front }} \\
0 & \text {, otherwise. }
\end{array}\right.
$$

\section{COMmunication FOR Human Robot InTERACTION}

Whenever a human operator is detected in the vicinity of the Tugger, the vehicle verbalizes its behavior so that the human operator can anticipate what the it will do. Specifically, the sign of the vector field $F(\phi)$ in the heading direction dynamics, Eq. 1, allows to predict if the vehicle will turn to the left, $F(\phi)>0$, or to the right, $F(\phi)<0$. The Tugger always avoids the human operators, but it may happen that a human operator actively comes very close to it $\left(d_{o b s h} \leq D_{h_{s} \text { stop }}\right)$. In such case, the velocity dynamics makes the vehicle to come to rest and the Tugger requests the human operator to move way. Given this rational, the messages to be sent to the human-robot interaction module (Fig. 5) are defined as follows:

$$
\begin{aligned}
& \operatorname{if}\left(c_{\text {human }}\right) \\
& \left\{\begin{array}{l}
\operatorname{if}\left(d_{\text {obsh }}>D_{h_{-} \text {stop }}\right) \\
\text { if }(F(\phi)<0) \\
\text { send }(\text { Attention! I'll overtake through the right }) \\
\text { elseif }(F(\phi)>0) \\
\text { send (Attention! I'll overtake through the left })
\end{array}\right. \\
& \text { else } \\
& \left\{\begin{array}{l}
\text { send(Please, get out of the way! } \\
\text { I can not get through. })
\end{array}\right.
\end{aligned}
$$

\section{IMPLEMENTATION}

Regarding the implementation, note that, $\phi(t)-\psi_{\text {obs }, i}=$ $-\theta_{s, i}$ in Eq. 5, is actually a constant, i.e. it is the inverse of the angle $\theta_{s, i}$ at which sector $i$ is centered relative to the forward direction of the Tugger. This is important because, for the implementation, it makes the collision avoidance behavior immune to errors in the calibration of the vehicle's heading direction in the world. Similarly, in Eq. 12, for the implementation one has $\phi(t)-\psi_{h, i}=-\theta_{o b s h, i}$, which is directly the bearing angle at which human $i$ is detected by the vision system (Fig. 4), and thus for the behavior of human avoidance the calibration of the vehicle's heading direction is irrelevant.

Equation 1 gives directly the angular velocity $\omega$. The Tugger's heading direction, $\phi$, is provided by the positioning system. Path velocity, $v$, is obtained applying the forward Euler method to Eq. 2. Applying the inverse kinematics model of the vehicle, one gets the values for the actuation variables (e.g., [3]): $\theta_{\text {steer }}=\tan ^{-1}\left(\omega L_{1} / v\right)$ and $v_{\text {steer }}=\sqrt{v^{2}+\omega^{2} L_{1}^{2}}$, where $L_{1}$ is the distance between the vertical axis of the steering wheel and the CRP. Parameters of the dynamics are tuned also to guarantee that $\left|\theta_{\text {steer }}\right|<\pi / 2$ and $v_{\text {steer }}<V_{\text {steer,max }}$.

\section{EXPERIMENTAL RESUlts}

In the following, we report the results of several experiments that highlight different features of the navigation system. In all the parameters are: $N$ sector $s=63, \Delta \theta_{s}=210 / N$ sectors $\mathrm{deg}, B=0.95 \mathrm{~m}, L_{1}=1.319 \mathrm{~m}, L_{2}=1.63 \mathrm{~m}, v_{\max }=0.5$, $k_{1,1}^{o b s}=1.0, k_{1,2}^{o b s}=1.2, k_{1,3}^{o b s}=1.5, k_{2,1}^{o b s}=0.75, k_{2,2}^{o b s}=0.8$, $k_{2,3}^{o b s}=1.5, \beta_{1}^{h}=1, \beta_{2}^{h}=2, \lambda_{v, t a r}=0.25, \lambda_{v, o b s}=0.25$, $\lambda_{v, \text { human }}=0.25, d_{\text {tar_stop }}^{C R P}=0.5 \mathrm{~m}, D_{\text {tar }}=3 \mathrm{~m}, D_{\text {obs }, \text { lat }}=$ $0.5 \mathrm{~m}, D_{\text {obs }, \text { front }}=2.5 \mathrm{~m}, D_{h_{-} \text {stop }}=1.5 \mathrm{~m}, D_{\text {obs }, h}=5 \mathrm{~m}$, $k_{\text {tar }}^{v}=3 \mathrm{~m}, k_{\text {obs }, 1}^{v}=10 \mathrm{~m}, k_{\text {obs }, 2}^{v}=2 \mathrm{~m}, k_{h}^{v}=3.5 \mathrm{~m}$.

\section{A. Experiment 1: Simulation of a typical Tugger operation}

In the first experiment it was tested the ability of the Tugger to safely navigate between pre-defined target via points, which are part of a typical mission/operation on a factory floor. Results are presented in simulation because it simplifies the simultaneous presentation of the behavioral dynamics that govern the behavior. The video showing a milkrun task can be seen in https://youtu.be/wlF29x3VEhw. The Tugger moves to the position given by each of the target via points, avoiding obstacles, and also avoiding a human operator walking around in the corridor while the Tugger is passing. The graphs shown in the video frames represent the individual contributions $f_{\text {tar }}, f_{\text {obs }}$ and $f_{\text {human }}$ - and their integration for the behavioral dynamics of the heading direction as defined by Eq. 1. Two particular situations are presented in Figs. 6 and 7. Parameters

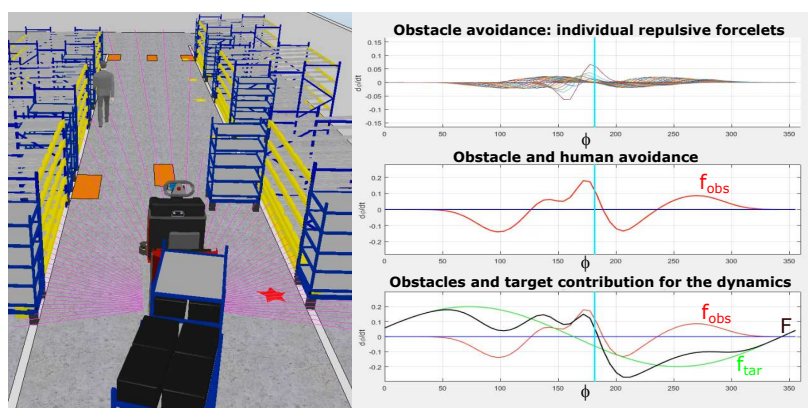

Fig. 6. Experiment 1: Left: Snapshot of the particular case in the moment the Tugger detects an obstacle, i.e. a shelf, in the path (human is too far away). Right: plot of the dynamics for this specific situation: $1^{\text {st }}$ plot individual repulsive force-lets from the detected obstructions, $f_{o b s, i}(\phi) ; 2^{\text {nd }}$ plot - the sum of all individual repulsive force-lets, $f_{o b s}(\phi) ; 3^{\text {rd }}$ plot - resultant dynamics, $F(\phi)=f_{o b s}(\phi)+f_{\text {tar }}(\phi)$. The current value of the Tugger's heading direction (indicated by the vertical cyan line) is very close to the attractor located near $185 \mathrm{deg}$ of the resultant dynamics.

have been tuned so that the heading direction, $\phi(t)$, follows very closely one of the attractors of the resultant dynamics most of the time. This can be seen in Fig. 8.

\section{B. Experiment 2: Tugger operation challenged by an abrupt perturbation}

In the second experiment it was tested the ability of the Tugger to handle unexpected events during task execution, 

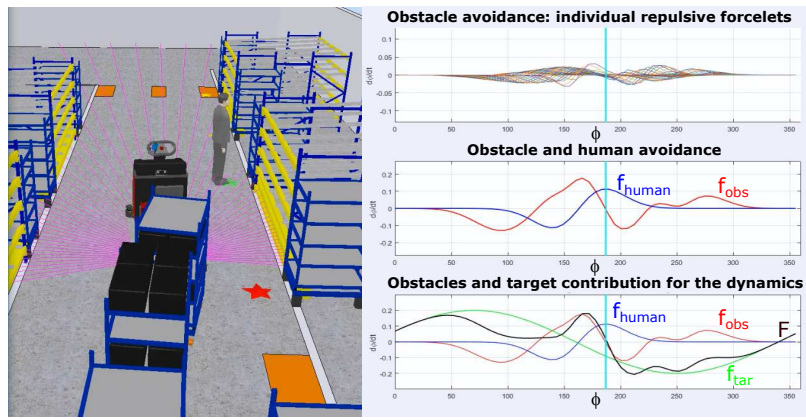

Fig. 7. Experiment 1: Left: Snapshot of the particular case in the moment the Tugger also detects the human operator. Right: plot of the dynamics for this specific situation: $1^{\text {st }}$ plot - individual repulsive force-lets from the detected obstructions, $f_{\text {obs }, i}(\phi) ; 2^{\text {nd }}$ plot - the sum of all individual repulsive forcelets, $f_{o b s}(\phi)$, and the repulsive force-let, $f_{\text {human }}(\phi)$ created by the detection of the human operator gives rise to the emergence of a repeller near 165 deg; $3^{r d}$ plot - resultant dynamics, $F(\phi)=f_{\text {obs }}(\phi)+f_{\text {human }}(\phi)+f_{\text {tar }}(\phi)$. The current value of the Tugger's heading direction (indicated by the vertical cyan line) is in the attractor near $190 \mathrm{deg}$ of the resultant dynamics.

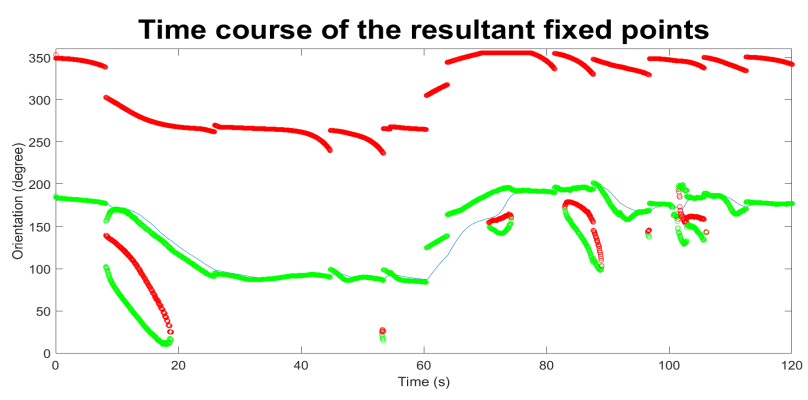

Fig. 8. Experiment 1: Time course of the resultant fixed points - attractors as green circles and repellers as red crosses - of the heading directions dynamics, Eq. 1, and the heading direction, $\phi(t)$ (blue line). As the Tugger moves and/or sensory information changes the attractors shift pulling the heading direction, $\phi$, along. Most of the time, $\phi(t)$ is in, or very close, to one of the moving attractors. Exception occurs when a bifurcation takes place (e.g., near $t=60 \mathrm{sec}, t=75 \mathrm{sec}$ ), the (moving) attractor the system was in disappears, and the heading direction needs some time to relax to a new attractor. (https://youtu.be/wlF29x3VEhw).

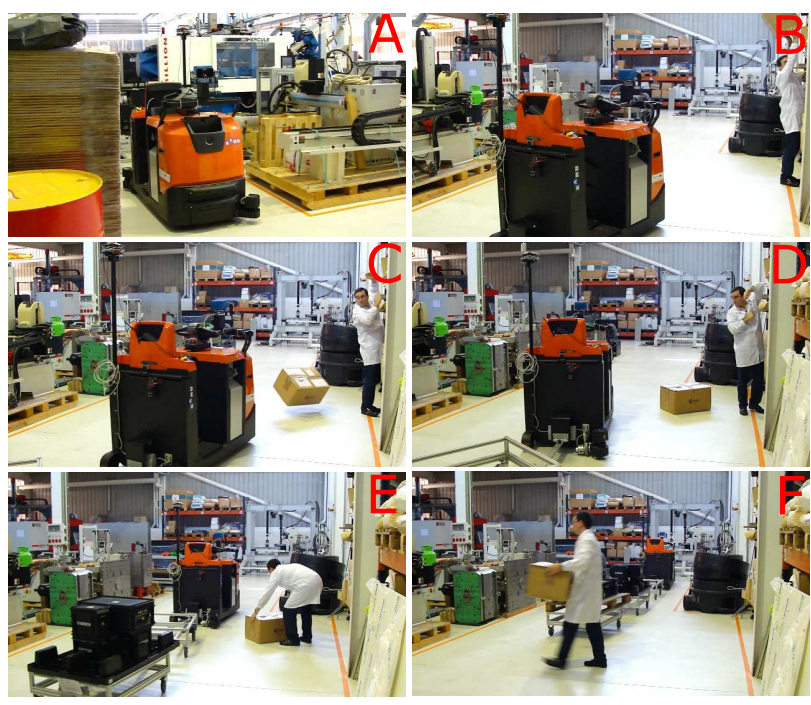

Fig. 9. Experiment 2: Tugger operation challenged by an abrupt perturbation. (https://youtu.be/hP9MNoBOhf8). such as the appearance of a sudden obstacle in its path. As it can be seen in Fig. 9, during the execution of its mission the Tugger is faced with the sudden appearance of a box that is dropped by a human operator (snapshots $\mathrm{A} \rightarrow \mathrm{C}$ ). This event challenges the vehicle's behavior/movement. The vehicle is able to avoid the collision with this sudden obstacle (snapshots $\mathrm{C} \rightarrow \mathrm{D}$ ), i.e. the vehicle deviates from it by turning left, then continues moving toward the target via point as soon as possible (snapshots $\mathrm{E} \rightarrow \mathrm{F}$ ).

C. Experiment 3: Tugger operation challenged by the coexistence of human operators - moving obstacles and verbal HRI

We have tested the capability of the Tugger to share the environment with human operators that may appear on its path while executing its mission. It is important that the vehicle senses their presence and safely avoid them, combining verbal interaction and appropriate motor behavior, while continuing the mission. As illustrated in Fig. 10, during the execution

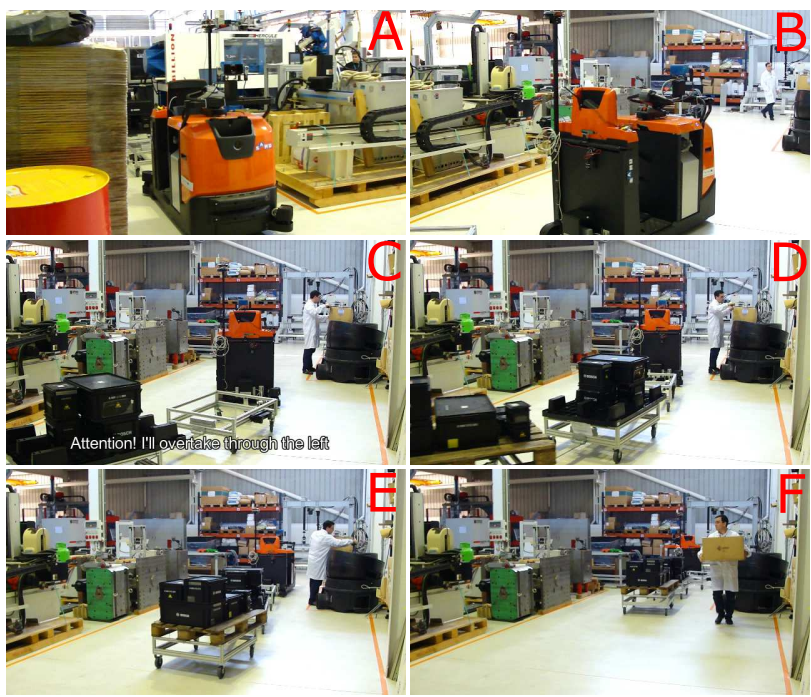

Fig. 10. Experiment 3: Tugger operation challenged by the coexistence of human operators - moving obstacles and verbal HRI. (https://youtu.be/FYiHtGG_dJQ).

of its mission the Tugger detects a human operator on its way (snapshots $\mathrm{A} \rightarrow \mathrm{B}$ ), and soon after adapts its behavior appropriately, i.e. it deflects and communicates to the human operator that it will overtake it through the left (snapshots $\mathrm{C} \rightarrow \mathrm{E}$ ). After avoiding the human operator, the vehicle returns to its route toward the target via point (snapshots $\mathrm{E} \rightarrow \mathrm{F}$ ).

D. Experiment 4: Vehicle operation challenged by human operators that defy mission accomplishment-conflict resolution through verbal HRI

In this experiment (Fig. 11) it was once again tested the capability of the Tugger to share the environment with human operators, but with the difference that this time the human operator defies mission accomplishment. The vehicle encounters a human operator and (as before) warns that it will overtake him (snapshots $\mathrm{A} \rightarrow \mathrm{C}$ ). However, the human operator moves toward the front of the vehicle blocking its movement 
(snapshots $\mathrm{D} \rightarrow \mathrm{F}$ ). The Tugger is therefore forced to stop, and reacts by demanding the person to leave the front (snapshot F). As soon as the human operator leaves the front (snapshot $\mathrm{K})$, the Tugger moves again, completing the task (snapshots $\mathrm{G} \rightarrow \mathrm{H})$.

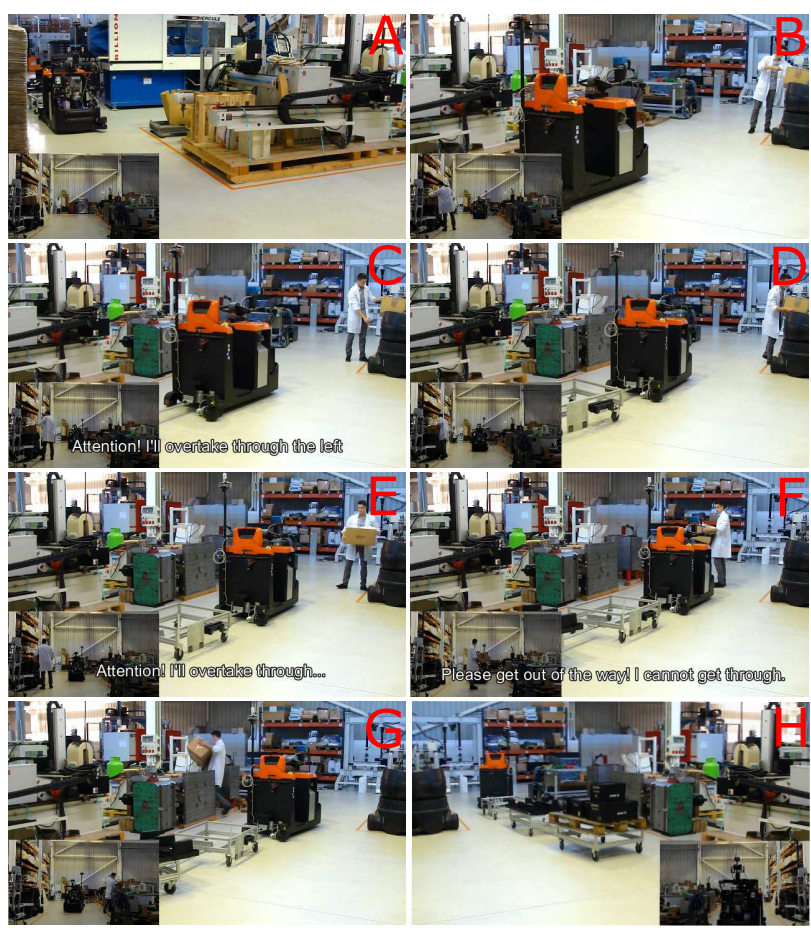

Fig. 11. Experiment 4: Vehicle operation challenged by human operators that defy mission accomplishment-conflict resolution through verbal HRI. (https://youtu.be/QewN1aHxW-Y).

\section{CONCLUSION}

We have presented a motion controller that generates collision free trajectories for autonomous Tugger vehicles operating in dynamic factory environments, where human operators and autonomous vehicles may coexist. The motion controller has been formalized as a dynamic system of path velocity and heading direction, whose vector fields change as sensory information varies. The parameters were tuned so that the control variables - path velocity and heading direction were close to an attractor of the resultant dynamics most of the time, because it contributes to make the system robust against perturbations. Several experiments - in simulation and real factory floor - have been reported to show different innovative features of the navigation system: flexible and safe solutions for human-aware autonomous navigation in dynamic and cluttered environments. This means, besides generating online collision free trajectories between target points, the system detects the presence of humans, interact with them showing awareness of their presence, and generate adequate motor behavior. The Tugger verbally interacts with human operators. This is very useful because it allows a human operator to predicted what the machine will do next. Future work concerns the implementation of controllers for docking operations of the Tugger and the chain of traillers, and motion control solutions for autonomous Stacker vehicles in pick and place operations, in dynamic environments, where different autonomous vehicles and humans share the workspace.

\section{REFERENCES}

[1] E. Bicho, P. Mallet, and G. Schöner, "Target representation on an autonomous vehicle with low-level sensors," The International Journal of Robotics Research, vol. 19, no. 5, pp. 424-447, May 2000.

[2] L. Bushnell, B. Mirtich, A. Sahai, and M. Secor, "Off-tracking bounds for a car pulling trailers with kingpin hitching," in Proc. of 1994 33rd IEEE Conference on Decision and Control, vol. 3. IEEE, 1994, pp. 2944-2949.

[3] Y. Kanayama, "Two dimensional wheeled vehicle kinematics," in Proc. of the 1994 IEEE Intl Conf on Robotics and Automation. IEEE, 1994, pp. 3079-3084.

[4] A. Kelly, B. Nagy, D. Stager, and R. Unnikrishnan, "Field and service applications-an infrastructure-free automated guided vehicle based on computer vision-an effort to make an industrial robot vehicle that can operate without supporting infrastructure," IEEE Robotics \& Automation Magazine, vol. 14, no. 3, pp. 24-34, 2007.

[5] T. Machado, T. Malheiro, S. Monteiro, W. Erlhagen, and E. Bicho, "Attractor dynamics approach to joint transportation by autonomous robots: theory, implementation and validation on the factory floor," Autonomous Robots, vol. 43, no. 3, pp. 589-610, 2019.

[6] H. Martínez-Barberá and D. Herrero-Pérez, "Autonomous navigation of an automated guided vehicle in industrial environments," Robotics and Computer-Integrated Manufacturing, vol. 26, no. 4, pp. 296-311, 2010.

[7] Z. Miljković, N. Vuković, M. Mitić, and B. Babić, "New hybrid visionbased control approach for automated guided vehicles," The International Journal of Advanced Manufacturing Technology, vol. 66, no. 1-4, pp. 231-249, 2013.

[8] S. Monteiro and E. Bicho, "Attractor dynamics approach to formation control: theory and application," Autonomous Robots, vol. 29, pp. 331$355,2010$.

[9] Omg.org, "About the unified modeling language specification version 2.5," https://www.omg.org/spec/UML/2.5, 2019, [Online; accessed 3 Apr. 2019].

[10] D. Pereira, J. Machado, and J. P. Mendonça, "Innovative coupling system for industrial vehicles: An agv oriented approach and development a new coupling system for improving trailer trajectory accuracy of agv vehicles," in 2017 Intl Conf on Engineering, Technology and Innovation, Funchal, Portugal, 27-29 June 2017, pp. 1145-1153.

[11] C. Ramer, J. Sessner, M. Scholz, X. Zhang, and J. Franke, "Fusing low-cost sensor data for localization and mapping of automated guided vehicle fleets in indoor applications," in 2015 IEEE Intl Conf on Multisensor Fusion and Integration for Intelligent Systems (MFI). IEEE, 2015, pp. 65-70.

[12] D. Ronzoni, R. Olmi, C. Secchi, and C. Fantuzzi, "Agv global localization using indistinguishable artificial landmarks," in 2011 IEEE International Conference on Robotics and Automation. IEEE, 2011, pp. 287-292.

[13] C. Rösmann, W. Feiten, T. Wösch, F. Hoffmann, and T. Bertram, "Trajectory modification considering dynamic constraints of autonomous robots," in ROBOTIK 2012; 7th German Conference on Robotics. VDE, 2012, pp. 1-6.

[14] L. Sabattini, V. Digani, C. Secchi, G. Cotena, D. Ronzoni, M. Foppoli, and F. Oleari, "Technological roadmap to boost the introduction of agvs in industrial applications," in 2013 IEEE 9th Intl Conf on Intelligent Computer Communication and Processing (ICCP). IEEE, 2013, pp. 203-208.

[15] G. Schöner, M. Dose, and C. Engels, "Dynamics of behavior: Theory and applications for autonomous robot architectures," Robotics and Autonomous Systems, vol. 16, pp. 213-245, 1995.

[16] M. Seder and I. Petrovic, "Dynamic window based approach to mobile robot motion control in the presence of moving obstacles," in Proc 2007 IEEE Intl Conf on Robotics and Automation. IEEE, 2007, pp. 19861991.

[17] TensorFlow, “Tensorflow," https://www.tensorflow.org, 2019, [Online; accessed 4 Apr. 2019].

[18] I. F. Vis, "Survey of research in the design and control of automated guided vehicle systems," European Journal of Operational Research, vol. 170, no. 3, pp. 677-709, 2006. 